\title{
TNF-a/LL-2 ratio discriminates latent from active tuberculosis in immunocompetent children: a pilot study
}

\author{
Nadège Gourgouillon', Agathe de Lauzanne22, Charles-Henry Cottart ${ }^{3}$, Emmanuel Curis ${ }^{4}$, Camille Debord', \\ Valérie Guérin-El Khourouj', Béatrice Pédron', Albert Faye ${ }^{2}$ and Ghislaine Sterkers ${ }^{1}$
}

\begin{abstract}
BACKGROUND: Distinguishing latent tuberculosis (LTB) from tuberculosis (TB) disease may be challenging in children. Here, we analyzed cytokine profiles that can distinguish the two infection stages in a nonendemic country (France).
\end{abstract}

METHODS: Immunocompetent children with LTB $(n=6)$ or TB disease $(n=8)$ (median age: 6.2 and 5.7 years, respectively) were analyzed. Four young uninfected children were included as controls. A Luminex assay evaluated cytokine responses to Mycobacterium tuberculosis antigens.

RESULTS: Poor interleukin-4 (IL-4) and IL-10 responses precluded analysis of these cytokines. Interferon- $\gamma($ IFN- $\gamma)$, tumor necrosis factor-a (TNF-a), IL-2, and T-helper type 1 (Th1) cytokines and IL-5, IL-13, T-helper type 2 (Th2) cytokines were simultaneously induced by antigens in 14/14 infected but 0/4 uninfected children. Th1 cytokine levels were similar in LTB and TB disease: IFN- $\gamma:$ 12,254 and 10,495 pg/ml; IL-2: 2,097 and $1,869 \mathrm{pg} / \mathrm{ml}$; and TNF-a: 1,020 and $2,875 \mathrm{pg} / \mathrm{ml}$, respectively. Th2 cytokine levels were similar and even higher in LTB than in TB disease: IL-5: 23 and $10 \mathrm{pg} / \mathrm{ml}$; IL-13: 284 and $109 \mathrm{pg} / \mathrm{ml}$, respectively. Positive correlation of cytokine levels, whether Th1 or Th2, was observed. Higher $(P=0.008)$ TNF-a/LL-2 ratios distinguished $6 / 8$ active TB disease cases from 6/6 LTB cases.

CONCLUSION: TNF-a/IL-2 ratio may discriminate TB disease from LTB in immunocompetent children. Larger studies in TB endemic settings must verify these results.

$\mathrm{T}$ uberculosis (TB) ranks among the world's top 10 causes of mortality. Approximately one-third of the world's population is infected with Mycobacterium tuberculosis. In 2010, 8.8 million incident cases of TB and 1.45 million deaths from TB were registered (1). The risk of progression to TB disease following primary infection is high in infants (20-40\%), slowly decreasing thereafter $(10-25 \%$ and $10 \%$ in young children and adolescents, respectively). The remaining infected children develop latent TB (LTB) (2). Of key importance, appropriate patient management to distinguish children with LTB from those with active early disease may be challenging. The main objective of this study was to determine whether certain cytokine profiles could distinguish LTB from TB disease.

The immune parameters responsible for progression and latency remain largely unknown. Immune responses to $M$. tuberculosis involve T-helper type 1 (Th1) cells that produce tumor necrosis factor- $\alpha$ (TNF- $\alpha$ ) and interferon- $\gamma$ (IFN- $\gamma$ ). A role for TNF- $\alpha$ in protection against $M$. tuberculosis has been definitely demonstrated by the higher rate of LTB reactivation following treatment with specific TNF- $\alpha$ inhibitors (3). IFN- $\gamma$ responses to $M$. tuberculosis antigens, selectively observed in individuals with TB infection, led to the development of IFN- $\gamma$ release assays for diagnosing $M$. tuberculosis infection (4). A role for IFN- $\gamma$ in conferring protection against $M$. tuberculosis has been demonstrated by the occurrence of severe mycobacterial disease in children with a mutation in the IFN$\gamma$-receptor gene (5). The role of T-helper type 2 (Th2) cells, which produce interleukin-4 (IL-4), IL-5, IL-10, and IL-13, is debatable. It has been suggested that Th2 secretion reflects an inappropriate response to mycobacteria. Indeed, the high percentages of IL-4-secreting T cells observed soon after $M$. tuberculosis infection could be associated with later development of TB disease, whereas long-term control of latent TB could be associated with optimal Th1 responses but low or even undetectable Th2 cytokine production (6). In early life, the high risk of progression to TB disease could also be associated with a bias toward low Th1 levels related to Th2 levels of cytokine responses to mycobacteria, especially in developing countries (7). Although less information is available regarding IL-2 responses, recent studies showing a switch from Th1 cells secreting IFN- $\gamma$ to T cells secreting both IL-2 and IFN- $\gamma$ following tuberculosis treatment, suggest a role for IL-2 in $M$. tuberculosis control $(8,9)$.

Although Th1 and Th2 cytokine responses to Bacille Calmette Guerin (BCG) vaccination can be detected even in infants, studies on cytokine responses to $M$. tuberculosis in children are scarce, especially in developed countries $(7,10)$. As far as we know, no study has analyzed cytokine profiles that can distinguish TB disease from TB infection. Here, the

\footnotetext{
'Laboratory of Immunology, Robert Debré Hospital, AP-HP-Faculty of Medicine, Paris 7 Denis Diderot University, Paris, France; ${ }^{2}$ Department of Pediatrics, Robert Debré Hospital, AP-HP-Faculty of Medicine, Paris 7 Denis Diderot University, Paris, France; ${ }^{3}$ Department of Biochemistry and Hormonology, Charles Foix Hospital, AP-HP-EA4466, Paris 5 Descartes University, Paris, France; ${ }^{4}$ Laboratory of Biomathematics, Faculty of Pharmacy Paris 5, Descartes University, Paris, France. Correspondence: Ghislaine Sterkers (ghislaine.sterkers@rdb.aphp.fr)
} 
patterns of cytokine production in the two stages of TB infection were compared in children.

Diagnosis of $M$. tuberculosis infection remains complex in children and requires clinical, radiological, histopathological, and immunological parameters as bacteriological confirmation is not the rule (11). The recently developed QuantiFERON-TB Gold In-Tube (QF-TB-IT) enzyme-linked immunosorbent assay measures IFN- $\gamma$ responses to the early secretory antigenic target-6, culture filtrate protein-10, and tuberculosis 7.7 (TB.7.7) antigens, which are present in M. tuberculosis complex but absent from all strains of Mycobacterium bovis BCG and almost all environmental mycobacteria. We previously reported that this assay has slightly lower sensitivity but higher specificity than the tuberculin skin test (TST) in children in France, where there is a low incidence of TB (12). As culture-confirmed TB disease is not the rule in pediatric TB and as there is no gold standard for the diagnosis of LTB, both TST and QF-TB-IT were

Table 1. Main characteristics of the studied population

\begin{tabular}{|c|c|c|c|}
\hline Variable & $\begin{array}{c}\text { Uninfected } \\
\text { contact }(n=4)\end{array}$ & $\operatorname{LTB}(n=6)$ & $\begin{array}{l}\text { TB disease } \\
(n=8)\end{array}$ \\
\hline Gender: male/female & $1 / 3$ & $5 / 1$ & $3 / 5$ \\
\hline $\begin{array}{l}\text { Age: median in years } \\
\text { (range) }\end{array}$ & $4.6(1.1-14.3)$ & $6.2(1.5-10.6)$ & $5.7(0.3-14.4)$ \\
\hline BCG vaccination & $4 / 4$ & $6 / 6$ & $6 / 8$ \\
\hline \multicolumn{4}{|l|}{$\begin{array}{l}\text { Recent ( } \leq 3 \text { months) } \\
\text { contact }\end{array}$} \\
\hline Documented & $4 / 4$ & $6 / 6$ & $7 / 8$ \\
\hline Presumed & & & $1 / 8$ \\
\hline TST positivity & $0 / 4$ & $6 / 6$ & $8 / 8$ \\
\hline QF-TB-IT positivity & $0 / 4$ & $6 / 6$ & $8 / 8$ \\
\hline
\end{tabular}

BCG, Bacille Calmette Guerin; LTB, latent tuberculosis; QF-TB-IT, QuantiFERON-TB Gold In-Tube test; TB, tuberculosis; TST, tuberculin skin test. used to denote or exclude $M$. tuberculosis infection in children involved in this study. Furthermore, children with underlying diseases or immune deficiency (confirmed or suspected) were carefully excluded for homogeneity purposes. Cytokines were measured in residual supernatants from QF-TB-IT assay.

\section{RESULTS}

We evaluated Th1 and Th2 cytokine responses in six children presenting LTB and in eight children presenting TB disease. Children were selected according to the following criteria: (i) similar age; (ii) similar exposure to M. tuberculosis (i.e., contact with an infectious individual within the 3 months preceding presentation); (iii) no known or suspected immune deficiency; (iv) absence of treatment; and (v) positive QF-TB-IT and TST tests. These rigid and conservative criteria were chosen to exclude procedural nonuniformity as far as possible. Also, distinguishing LTB from TB disease may be especially difficult in this population. Four age-matched healthy children with recent $\mathrm{TB}$ contact but who were uninfected were included as a reference group. The main characteristics of the groups are shown in Table 1. IFN- $\gamma$-responses were evaluated using both the QF-TB-IT and Luminex assays, whereas the other cytokines were evaluated using only the Luminex assay.

As expected, uninfected children in whom the QF-TB-IT test was negative (Table 1 ) had no IFN- $\gamma$ response to $M$. tuberculosis antigens in the multiplex assay (ratio of values in stimulated/ unstimulated cultures ranging from 0.50 to 0.70 in Figure 1). In contrast, all the infected children who were selected according to QF-TB-IT positivity (Table 1 ) demonstrated IFN- $\gamma$ responses (ratio of values in stimulated/unstimulated cultures ranging from 8.9 to 9,500 ) (Figure 1).

Early secretory antigenic target-6, culture filtrate protein-10, and TB7.7 antigens, which do not cross-react with BCG or mycobacteria other than $M$. tuberculosis, did not induce a

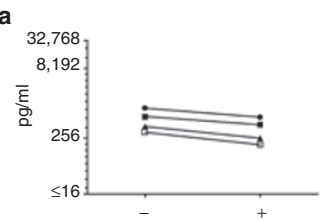

b
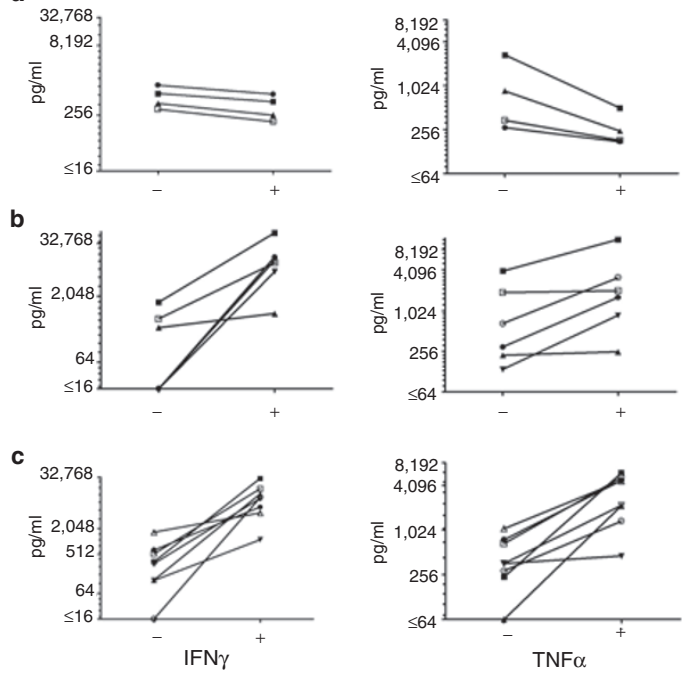
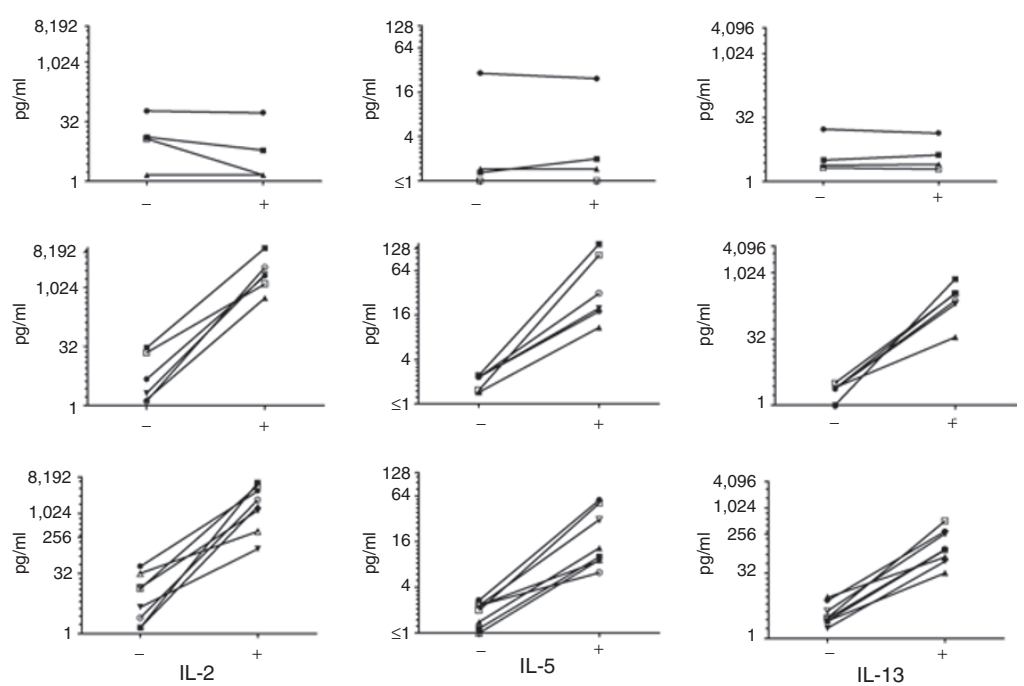

Figure 1. T-helper type 1 and T-helper type 2 responses to Mycobacterium tuberculosis antigens in latent tuberculosis (LTB) and tuberculosis (TB) disease. Blood samples from controls (a), children with LTB $(\mathbf{b})$ and children with TB disease (c) were stimulated with a mixture of early secretory antigenic target-6, culture filtrate protein-10, and TB7.7 proteins antigens (+) or not (-). Individual changes in values from unstimulated cultures (left symbols) to stimulated cultures with $M$. tuberculosis antigens (right symbols) are reported. $P$ values (Wilcoxon test (W) or Student's $t-$ test ( $T$ )) between stimulated and nonstimulated cultures range from 0.02 to 0.031 in (b) and 0.004 to 0.018 in (c). IFN, interferon; IL, interleukin; TNF, tumor necrosis factor. 

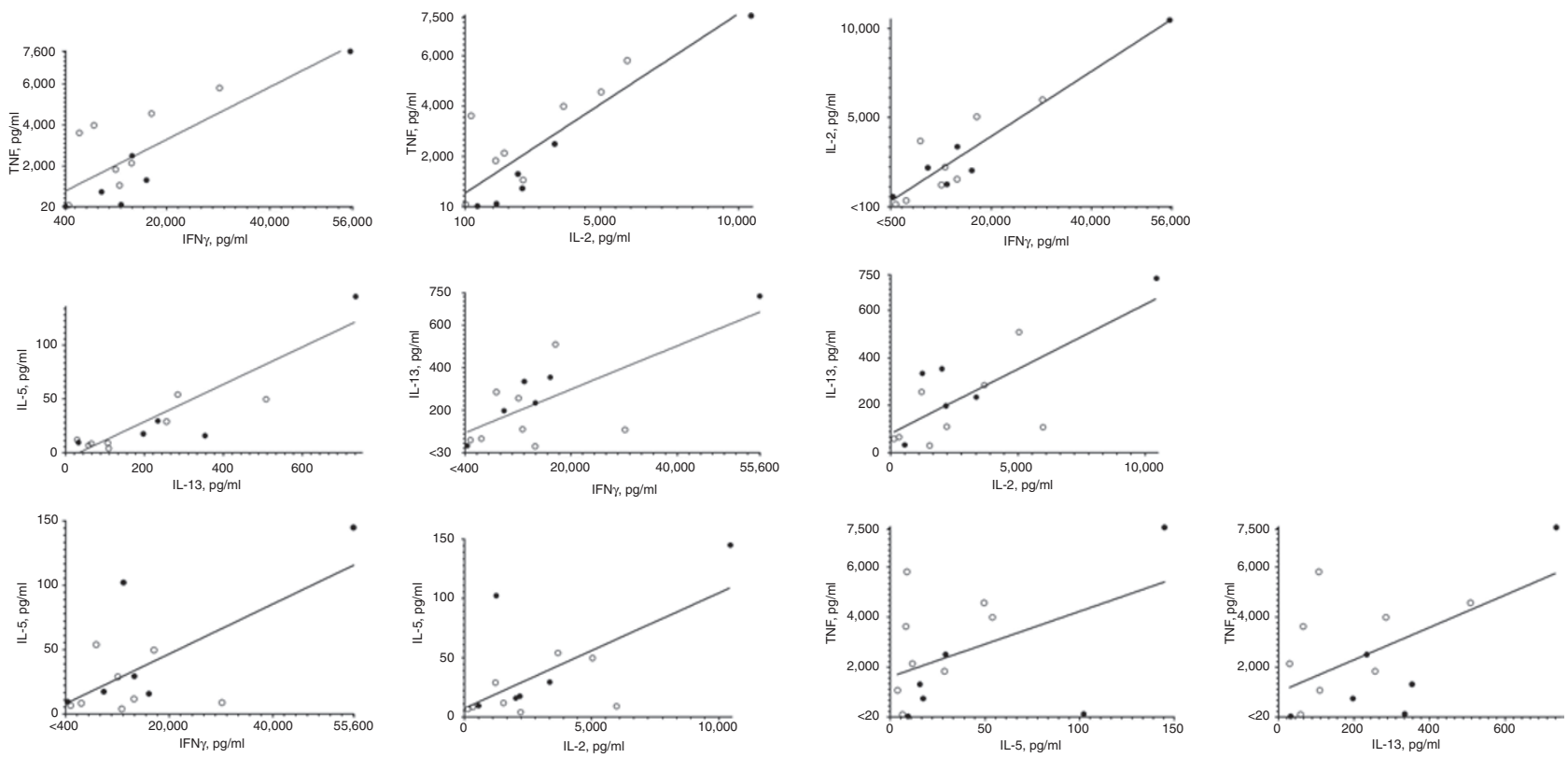

Figure 2. Positive correlations between T-helper type 1 and T-helper type 2 responses in latent tuberculosis (LTB) and tuberculosis (TB) disease, but a higher tumor necrosis factor-a/interleukin-2 (TNF- $\alpha$ /IL-2) ratio is seen in TB disease. Blood samples from children with LTB and from children with TB disease were in vitro stimulated or not with early secretory antigenic target-6, culture filtrate protein-10, and TB7.7 antigens. Individual values in stimulated cultures minus values in nonstimulated cultures are reported in LTB (filled circles) and TB disease (open circles). Correlations between various values were assessed by calculation of Spearman's rank correlations. Linear regression was used to estimate association between cytokines and to assess cytokine profiles. $P$ values were $<0.005$ for TNF- $\alpha /$ IFN- $\gamma$, TNF- $\mathrm{a} / \mathrm{IL}-2, \mathrm{IL}-2 / \mathrm{IFN}-\gamma$, and IL-5/IL-13. $P$ values were $<0.05$ for IL-13/IFN- $\gamma$ and IL-13/IL-2. $R$ was 0.66 for TNF- $\mathrm{a} /$ IFN- $\gamma ; 0.74$ for TNF- $\alpha / I L-2 ; 0.77$ for IL-2/IFN- $\gamma ; 0.77$ for IL-5/IL-13; 0.57 for IL-13/IFN- $-; 0.56$ for IL-13/IL-2; 0.42 for IL-5/IFN- $;$; 0.47 for IL-5/IL-2; 0.36 for TNF-a/ IL-5; and 0.41 for TNF-a/IL-13. IFN, interferon; IL, interleukin.

secretion of the other cytokines in uninfected children. In contrast, $M$. tuberculosis antigens induced IL-2 and TNF- $\alpha$ secretion in TB disease ( $P=0.012$ for IL-2 and TNF- $\alpha$ ) and in LTB $(P=0.019$ for IL-2 and TNF- $\alpha$ ) (Figure 1$)$. The intensity levels of IL-2 in stimulated cultures were similar in LTB and TB disease: i.e., 2,097 and $1,869 \mathrm{pg} / \mathrm{ml}(P=0.852)$, respectively. TNF- $\alpha$ levels were higher $(P=0.019)$ in TB disease (median: $2,875 \mathrm{pg} / \mathrm{ml}$ ) than in LTB (median: $1,020 \mathrm{pg} / \mathrm{ml}$ ).

Poor IL-4 responses (ratio of the values in stimulated/ unstimulated cultures $<2$ in $2 / 4$ patients with $\mathrm{TB}$ disease and $3 / 3$ patients with LTB in our analysis) and undetectable IL-10 responses precluded analysis of these two cytokines. The two other Th2 cytokines included in the study were, however, induced by $M$. tuberculosis antigens in infected children (Figure 1). The intensity levels of IL-5 and IL-13 secretion in stimulated cultures were similar and even higher in LTB than in TB disease. IL-5 median levels were $23 \mathrm{pg} / \mathrm{ml}$ for LTB and 10 $\mathrm{pg} / \mathrm{ml}$ for TB disease $(P=0.142)$, and IL-13 median levels were $284 \mathrm{pg} / \mathrm{ml}$ for LTB and $109 \mathrm{pg} / \mathrm{ml}$ for TB disease $(P=0.284)$.

As shown in Figure 2, the intensity levels of IFN- $\gamma$ and TNF- $\alpha$ Th1 responses positively correlated with each other and with IL-2. A positive correlation between Th2 cytokine levels (IL-5/IL-13) and between IFN- $\gamma$ (Th1) and IL-13 or IL-5 levels was also observed.

The distribution of individual cytokine values in TB disease and LTB was subsequently analyzed. Among all principal component analysis linear combinations of the cytokine levels, only the line for the TNF- $\alpha /$ IL-2 couple permitted discrimination between patients with TB disease and those with LTB, with 6/8 values from patients with TB disease over the line, but $0 / 6$ values from children with LTB. Using this as a classifier leads to classification that is significantly better than random ( $P=0.01$, Fisher's exact test).

Further simplification consisted in using the TNF- $\alpha /$ IL-2 ratio, which was significantly different between patients with LTB and those with TB disease $(P=0.008)$. Using this ratio as a classifier generated the receiver operating characteristic curve that appears in Figure 3. This figure suggests a cutoff at around 0.73 (i.e., if the TNF- $\alpha / \mathrm{IL}-2$ ratio is $<0.73$, the patient is classified as having LTB, otherwise as having TB disease), with $88 \%$ sensitivity and $83 \%$ specificity (Youden's index: $J=0.71$ ). Using this cutoff, classification is significantly better than random $(P=0.026$, Fisher's exact test $)$.

\section{DISCUSSION}

Although cytokine analysis has greatly contributed to the understanding of TB pathogenesis, data on cytokine profiles that might distinguish progression from latency of TB infection are scarce and even controversial (13-16). Furthermore, most studies in this field were performed in individuals more than 15 years old. Assessments of the more vulnerable pediatric population remains to be performed.

Background levels of cytokines were observed in unstimulated samples from most children whether infected with $M$. tuberculosis or not. As previously noticed by others, even higher analyte levels may be found in unstimulated than in stimulated QF-TB-IT samples in noninfected controls $(17,18)$. 


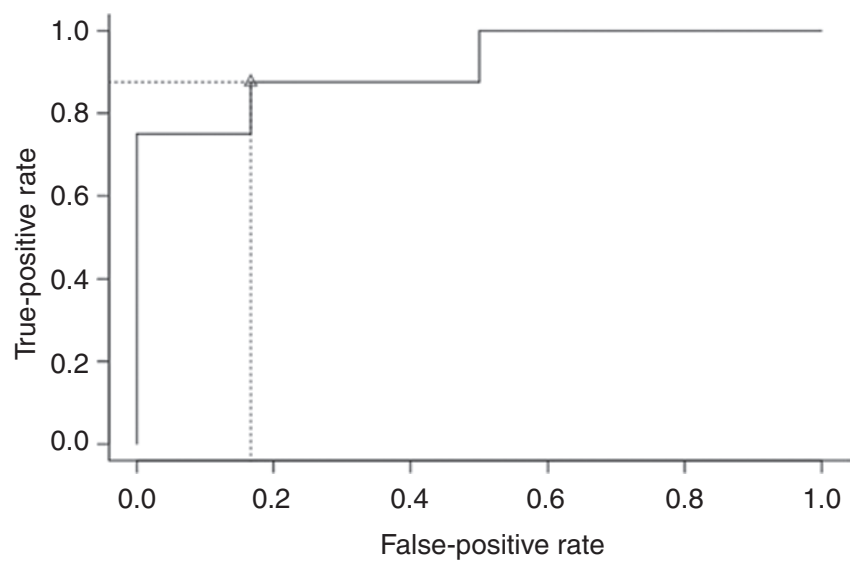

Figure 3. Receiver operating characteristic curve for the use of the tumor necrosis factor-a/interleukin-2 ratio as a classifier to distinguish between latent tuberculosis and tuberculosis disease. As detailed in the Results section, sensitivity $=0.83$ and specificity $=0.88$. Dashed lines indicate prediction of true-positive (horizontal) and false-positive (vertical) rates.

The mechanisms involved in this process remain to be clarified, but similar background levels were observed in infected and noninfected children. In stimulated cultures, the intensity levels of IFN- $\gamma$ responses appeared highly similar in patients with LTB and those with TB disease: i.e., 12,254 and 10,494 $\mathrm{pg} / \mathrm{ml}(P=0.662)$, respectively. Similar observations have been found in adults (19). In addition, IL-4 and IL-10 were poorly induced by $M$. tuberculosis antigens in this study as previously observed in other developed countries (6) Our results, however, disagree with the paradigm that progressive TB is accompanied by decreased Th1 and/or increased Th2 expression (6). Indeed, two Th2 cytokines (IL-5 and IL-13) were induced at similar and even higher levels in LTB than in TB disease. At variance with our data, Lalor et al. recently reported that high Th1 responses were associated with high Th2 responses to mycobacteria antigens in infants following BCG vaccination (7). High levels of Th2 responses to mycobacteria antigens, in the context of protection against $M$. tuberculosis (BCG vaccination in the Lalor et al. study), and control of M. tuberculosis (LTB in this study) could therefore reflect downregulation of IFN- $\gamma$ inflammatory responses rather than susceptibility to disease progression.

More important, TNF- $\alpha / \mathrm{IL}-2$ ratio discriminated TB disease from LTB with higher TNF- $\alpha$ /IL-2 ratio in TB disease. This observation, made in young children, is at variance with several studies that reported an association between high TNF- $\alpha$ responses to $M$. tuberculosis and disease activity in adults. Sutherland et al. first reported a higher proportion of T cells producing TNF- $\alpha$ alone in TB disease (19). Very recently, Harari et al. even proposed that high levels of $M$. tuberculosis-specific $\mathrm{CD}^{+}$secreting TNF- $\alpha$ might be even used as a signature of TB disease (9).

Of note, the biomarker identified in Lalor's study requires using a highly sophisticated intracytoplasmic cytokine staining assay that is difficult to carry out in routine clinical applications. We think that the use of supernatants from the commercialized QF-TB-IT kit in a Luminex assay as in our study is far easier to assess and standardize.
The fundamental role of TNF- $\alpha$ in the control of M. tuberculosis infection is well established (3). Increased TNF- $\alpha$ levels therefore suggest an ongoing high inflammatory response rather than control of TB infection.

Our results also support the contention that $M$. tuberculosis in LTB in young age is associated with relatively higher IL-2 responses. In line with this observation, several recent studies have suggested an association between control of $M$. tuberculosis infection by anti-TB chemotherapy and an increased proportion of $\mathrm{CD}^{+}{ }^{+} \mathrm{T}$ cells specific for M. tuberculosis antigens that secrete IL-2 in LTB in adults $(8,9)$. Even detection of IL-2 in addition to IFN- $\gamma$ discriminated active TB from LTB in one study (20). A greater IL-2 response in controlled M. tuberculosis infection is consistent with the current paradigm of immunity to intracellular pathogens. In several viral models, $\mathrm{T}$ cells mostly produce IFN- $\gamma$, TNF- $\alpha$ or both during viral replication and active disease. In contrast, an increasing proportion of polyfunctional responses involving cells that produce both IL-2 and IFN- $\gamma$ is found in individuals who control the virus with no sign of clinical disease (21).

\section{CONCLUSION}

The limitations of the study are (i) the small sample size of a highly selected series of immunocompetent children and (ii) diagnosis of $M$. tuberculosis infection that was based on both TST and QF-TB-IT positivity following a recent contact with M. tuberculosis. This study, however, describes a new method to identify TNF- $\alpha$ /IL-2 ratios that may help in distinguishing LTB from TB disease in pediatric settings. Further studies will be necessary to determine how TNF- $\alpha / \mathrm{IL}-2$ ratios could also be used as biomarkers for the diagnosis and staging of $M$. tuberculosis infection in more heterogeneous populations and, if so, whether they could provide more accurate determination of the cutoff value.

\section{METHODS}

\section{Patients: Diagnosis and Rational for Inclusion Criteria}

Residual supernatants from all QF-TB-IT assays sequentially carried out in our pediatric center (Robert Debré Hospital, Paris, France) from January 2009 to May 2010 were prospectively conserved for use in this study following informed consent from the parents or guardians of the children and approval by the local ethic committee from Robert Debré Hospital, Paris, France. The six children who presented during this period with recent ( $\leq 3$ months) contact with $\mathrm{TB}$, without known or suspected immunodeficiency and with a final diagnosis of LTB were included. LTB was defined as a positive response to TST (i.e., $\geq 5 \mathrm{~mm}$ when nonvaccinated or $\geq 10 \mathrm{~mm}$ when $\mathrm{BCG}$ vaccinated with a history of TB contact or $\geq 15 \mathrm{~mm}$ when BCG vaccinated without a history of TB contact) and QF-TB-IT tests in exposed individuals but no clinical sign of active disease and a normal chest $\mathrm{X}$ ray. Among the patients who presented with TB disease at this same center during the same period, only the eight children with TB disease with similar age as patients with LTB, with recent ( $\leq 3$ months) close contact with TB and without known or suspected immunodeficiency were included. TB disease was defined as an active pulmonary disease confirmed by positive culture of M. tuberculosis from sputum specimens $(n=5)$ or extrapulmonary disease following contact with TB $(n=3)$ and a positive response to TST (according to the same criteria as LTB) and QF-TB-IT tests. Four healthy children with similar age as the children with LTB and those with TB disease who were not infected despite 
a recent ( $\leq 3$ months) close contact with TB were also evaluated as controls (TB infection was excluded by the absence of pathological clinical or chest $\mathrm{X}$ ray signs and negative responses to both TST and QF-TB-IT).

\section{QF-TB-IT}

The QF-TB-IT (Cellestis, Carnegie, Australia) test was carried out according to the manufacturer's instructions and as described earlier (12). Briefly, blood ( $1 \mathrm{ml}$ ) was drawn into each of three separate heparinized tubes: nil, mitogen control (phytohemagglutinin ), and M. tuberculosis peptides (early secretory antigenic target-6, culture filtrate protein-10, and TB7.7). The tubes were incubated at $37^{\circ} \mathrm{C}$ within $2 \mathrm{~h}$ of venipuncture. Plasma was harvested after $18-24 \mathrm{~h}$ of incubation for enzyme-linked immunosorbent assay IFN- $\gamma$ analysis with the reagents included in the kit. Results expressed as the concentration of IFN- $\gamma$ detected minus the concentration of IFN- $\gamma$ in the respective nil control plasma, $\geq 0.35 \mathrm{IU} / \mathrm{ml}$ were considered positive.

\section{Luminex Assay for Cytokines}

Concentrations of Th1 and Th2 cytokines were measured in residual plasma from the QF-TB-IT tubes containing or not containing early secretory antigenic target-6, culture filtrate protein-10, and TB7.7 M. tuberculosis antigens in a multiplexed microsphere-based assay (Luminex human custom made 7-plex cytokine kit; Bio-Rad, Hercules, CA) according to the manufacturer's instructions. Briefly, plasma was diluted one-fourth with sample diluent provided in the kit. After prewetting of the filter plate, $50 \mu \mathrm{l}$ of bead suspension was added to each well and washed twice with buffer. Fifty microliters of standards, controls, and samples were then added, sealed, and shaken for $30 \mathrm{~s}$ at $1,100 \mathrm{rpm}$, and incubated for $1 \mathrm{~h}$ at $300 \mathrm{rpm}$ at room temperature. Plates were washed and $25 \mu$ of detection antibody were added. After shaking, plates were incubated for $30 \mathrm{~min}$ at $300 \mathrm{rpm}$ in the dark. After washing, $50 \mu \mathrm{l}$ streptavidin-phycoerythrin was added to each well, and plates were sealed and shaken at 1,100 rpm for $30 \mathrm{~s}$, then at $300 \mathrm{rpm}$ for $10 \mathrm{~min}$ in the dark. Finally, plates were washed, mixed and immediately read on the Bioplex analyzer, using Bioplex manager software (version 5.0; Bio-Rad). A curve fit was applied to each standard according to the manufacturer's manual and sample concentrations were interpolated from the standard curves. All samples were assayed in duplicate or triplicate.

\section{Statistical Analysis}

Patient characteristics were reported as count or median (range). Statistical analyses were carried out using Graph Pad Prism software (version 5.00 for Windows; Graph Pad, San Diego, CA) and R (version 2.12.2 for Linux ) (22) with the additional package ROCR (23). All tests were two sided, with a type I error of 0.05 .

Differences in cytokine levels between nonstimulated and antigenstimulated cultures in each group were assessed using either the paired Student's $t$-test after assessment of normality (by the Shapiro-Wilk test, and graphically, by means of a quantile-quantile plot) or, when applicable $(n>3)$, the Wilcoxon signed-rank test (Figure 1$)$.

Correlationsbetween thedifferentcytokinelevels(antigen-stimulated minus nonstimulated cultures) measured by multiplex were assessed by calculating a Spearman's rank correlation.

Comparisons of cytokine levels among uninfected controls, patients with LTB, and those with TB were done by multiple Mann-Whitney tests, with $P$ values adjusted for multiplicity, using Holm's correction.

Principal component analysis was used to search for linear combinations of cytokine levels, permitting discrimination between patients with LTB and those with TB; such classifiers were then further simplified to ratios, and receiver operating characteristic curves were used to select the best cutoff for the ratio. Classification quality was assessed using Fisher's exact test for $2 \times 2$ contingency tables between the predicted and real status of the patients, and quantified according to various classical indexes.

\section{ACKNOWLEDGMENTS}

We thank Guylaine Boiry, Anne-Marie Courchinoux, Pricilia Egremonte, Elodie Geneletti, and Judith Tholle for excellent technical assistance.

\section{STATEMENT OF FINANCIAL SUPPORT}

This observational study was partly supported by Assistance Publique-Hôpitaux de Paris (AP-HP) funds.

\section{REFERENCES}

1. WHO report. Global tuberculosis control 2011. WHO, 2011. (http://www. who.int/tb/publications/global_report/2011/gtbr11_full.pdf.)

2. Feja K, Saiman L. Tuberculosis in children. Clin Chest Med 2005;26: 295-312, vii.

3. Stenger S. Immunological control of tuberculosis: role of tumour necrosis factor and more. Ann Rheum Dis 2005;64:Suppl 4:iv24-8.

4. Ferrara G, Losi M, D'Amico R, et al. Use in routine clinical practice of two commercial blood tests for diagnosis of infection with Mycobacterium tuberculosis: a prospective study. Lancet 2006;367:1328-34.

5. van de Vosse E, Hoeve MA, Ottenhoff TH. Human genetics of intracellular infectious diseases: molecular and cellular immunity against mycobacteria and salmonellae. Lancet Infect Dis 2004;4:739-49.

6. Rook GA. Th2 cytokines in susceptibility to tuberculosis. Curr Mol Med 2007;7:327-37.

7. Lalor MK, Smith SG, Floyd S, et al. Complex cytokine profiles induced by BCG vaccination in UK infants. Vaccine 2010;28:1635-41.

8. Wilkinson KA, Wilkinson RJ. Polyfunctional T cells in human tuberculosis. Eur J Immunol 2010;40:2139-42.

9. Harari A, Rozot V, Enders FB, et al. Dominant TNF-a+ Mycobacterium tuberculosis-specific $\mathrm{CD} 4+\mathrm{T}$ cell responses discriminate between latent infection and active disease. Nat Med 2011;17:372-6.

10. Soares AP, Scriba TJ, Joseph S, et al. Bacillus Calmette-Guérin vaccination of human newborns induces $\mathrm{T}$ cells with complex cytokine and phenotypic profiles. J Immunol 2008;180:3569-77.

11. Jasmer RM, Nahid P, Hopewell PC. Clinical practice. Latent tuberculosis infection. N Engl J Med 2002;347:1860-6.

12. Debord C, De Lauzanne A, Gourgouillon N, et al. Interferon-gamma release assay performance for diagnosing tuberculosis disease in 0 - to 5 -year-old children. Pediatr Infect Dis J 2011;30:995-7.

13. Al-Attiyah R, Madi N, El-Shamy AS, Wiker H, Andersen P, Mustafa A. Cytokine profiles in tuberculosis patients and healthy subjects in response to complex and single antigens of Mycobacterium tuberculosis. FEMS Immunol Med Microbiol 2006;47:254-61.

14. Ottenhoff TH, Verreck FA, Hoeve MA, van de Vosse E. Control of human host immunity to mycobacteria. Tuberculosis (Edinb) 2005;85:53-64.

15. Turner J, Gonzalez-Juarrero M, Ellis DL, et al. In vivo IL-10 production reactivates chronic pulmonary tuberculosis in C57BL/6 mice. J Immunol 2002;169:6343-51.

16. Lin PL, Flynn JL. Understanding latent tuberculosis: a moving target. J Immunol 2010;185:15-22.

17. Lighter-Fisher J, Peng CH, Tse DB. Cytokine responses to QuantiFERON ${ }^{\circ}$ peptides, purified protein derivative and recombinant ESAT-6 in children with tuberculosis. Int J Tuberc Lung Dis 2010;14:1548-55.

18. Chegou NN, Black GF, Kidd M, van Helden PD, Walzl G. Host markers in QuantiFERON supernatants differentiate active TB from latent TB infection: preliminary report. BMC Pulm Med 2009;9:21.

19. Sutherland JS, Adetifa IM, Hill PC, Adegbola RA, Ota MO. Pattern and diversity of cytokine production differentiates between $\mathrm{Myco}$ bacterium tuberculosis infection and disease. Eur J Immunol 2009; 39:723-9.

20. Biselli R, Mariotti S, Sargentini V, et al. Detection of interleukin-2 in addition to interferon-gamma discriminates active tuberculosis patients, latently infected individuals, and controls. Clin Microbiol Infect 2010; 16:1282-4.

21. Pantaleo G, Harari A. Functional signatures in antiviral T-cell immunity for monitoring virus-associated diseases. Nat Rev Immunol 2006;6:417-23.

22. Team RDC. A Language and Environment for Statistical Computing. R Fundation for Statistical Computing, Vienna, Austria, 2011. (http:// www.r-project.org/links.html).

23. Tobias J, Sander O, Beerenwinkel N, Lengauer RT. 2009. ROCR: Visualizing the performance of scoring classifiers. R package version 0.0-4. (http:// CRAN.R-project.org/package $=$ ROCR.). 